\title{
Biocompatibility of a self-assembled glycol chitosan nanogel
}

\author{
Paula Pereira ${ }^{a}$, Sílvia S. Pedrosa ${ }^{\mathrm{a}}$, Alexandra Correia ${ }^{\mathrm{b}}$, Cristovao F. Lima ${ }^{\mathrm{c}}$, Mercedes Peleteiro Olmedo ${ }^{\mathrm{d}}$, \\ África González-Fernández ${ }^{\mathrm{d}}$, Manuel Vilanova ${ }^{\mathrm{b}, \mathrm{e}}$, Francisco M. Gama ${ }^{\mathrm{a}, *}$ \\ ${ }^{a}$ Centre of Biological Engineering, University of Minho, Campus de Gualtar, 4710-057 Braga, Portugal \\ ${ }^{\mathrm{b}}$ IBMC - Institute of Molecular and Cell Biology, Rua Campo Alegre, 4099-003 Porto, Portugal

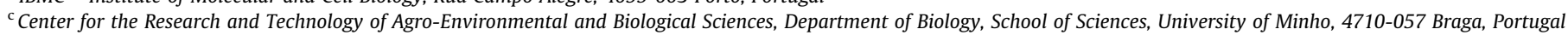 \\ ${ }^{\mathrm{d}}$ Immunology, Institute of Biomedical Research (IBIV), Biomedical Research Center (CINBIO), University of Vigo, 36310 Campus Lagoas de Marcosende, Vigo, Spain \\ ${ }^{\mathrm{e}}$ Abel Salazar Biomedical Sciences Institute, University of Porto, Rua de Jorge Viterbo Ferreira $n^{\circ} 228$, Porto 4050-313, Portugal
}

\section{A R T I C L E I N F O}

\section{Article history:}

Received 14 July 2014

Accepted 17 November 2014

Available online 4 December 2014

\section{Keywords:}

Glycol chitosan nanogel

Cytotoxicity

Apoptosis

Cell cycle

Complement activation

Haemocompatibility

\begin{abstract}
A B S T R A C T
The research of chitosan-based nanogel for biomedical applications has grown exponentially in the last years; however, its biocompatibility is still insufficiently reported. Hence, the present work provides a thorough study of the biocompatibility of a glycol chitosan (GC) nanogel. The obtained results showed that GC nanogel induced slight decrease on metabolic activity of RAW, 3T3 and HMEC cell cultures, although no effect on cell membrane integrity was verified. The nanogel does not promote cell death by apoptosis and/or necrosis, exception made for the HMEC cell line challenged with the higher GC nanogel concentration. Cell cycle arrest on G1 phase was observed only in the case of RAW cells. Remarkably, the nanogel is poorly internalized by bone marrow derived macrophages and does not trigger the activation of the complement system. GC nanogel blood compatibility was confirmed through haemolysis and whole blood clotting time assays. Overall, the results demonstrated the safety of the use of the GC nanogel as drug delivery system.
\end{abstract}

(c) 2014 Elsevier Ltd. All rights reserved.

\section{Introduction}

Nanoparticles have been largely researched as drug nanocarriers, yet their interaction with cells and extracellular environment is still poorly explored. The evaluation of the potential effects of drug delivery devices on the biological systems is indeed a crucial requirement in the development of nanomedicines: cytotoxicity, haematocompatibility (haemolysis and complement activation), inflammatory response; biodegradability and potential cytotoxicity of the degradation products, cellular uptake and intracellular fate, in vivo biodistribution studies, all assist in allowing a better definition of the biological properties of the novel polymers and their polymeric nanoparticles. (Gaspar and Duncan, 2009; Naahidi et al., 2013; Rodrigues et al., 2012) The so called "biocompatibility" is largely dependent on the physical and chemical properties of the nanoparticles (size, shape and surface characteristics), as well as on the used raw material (Liu et al., 2013; Naahidi et al., 2013).

\footnotetext{
* Corresponding author. Tel.: +351253604 418.

E-mail addresses: paulapereira@deb.uminho.pt (P. Pereira), silviasantospedrosa @deb.uminho.pt (S.S. Pedrosa), alexlopescorreia@gmail.com (A. Correia), lima@ bio.uminho.pt (C.F. Lima), mpeleteiro@uvigo.es (M.P. Olmedo), gonzalezfernande zafrica@gmail.com (Á. González-Fernández), vilanova@icbas.up.pt (M. Vilanova), fmgama@deb.uminho.pt (F.M. Gama).
}

The use of polymeric nanoparticles based on chitosan has been extensively reported in biomedical applications, due to its interesting characteristics. Non-toxicity, biocompatibility, biodegradability, antibacterial activity, mucoadhesiveness and permeation enhancing properties are among the features that have been reported, the cationic character being responsible for some of them (Bernkop-Schnurch and Dunnhaupt, 2012; Croisier and Jérôme, 2013). Although often claimed as biocompatible, more comprehensive studies are required for a proper understanding of the biological effects of this polymer and of its nanoformulations.

As reported previously, a polymeric nanoparticle made of glycol chitosan (GC), here designated GC nanogel, has been synthesized in our laboratory by chemical grafting hydrophobic chains on the GC backbone, yielding an amphiphilic polymer capable of self-assembling in aqueous environment (Pereira et al., 2013). The present work focuses on the detailed evaluation of the biocompatibility of this nanogel. For this purpose, in vitro cell toxicity of the GC nanogel was evaluated using the 3-(4,5-dimethylthiazol-2-yl)-2,5diphenyltetrazolium bromide (MTT) and lactate dehydrogenase (LDH) release assays to gauge the nanogel cytotoxicity. Induction of apoptosis and/or cell cycle arresting was tested through flow cytometry. The complement activation was semi-quantified by western blot, analysing the degradation of the C 3 factor. Interaction with murine macrophages was observed through confocal 
microscopy. Finally, the nanogel haemocompatibility was evaluated through haemolysis and whole blood clotting time assays.

\section{Experimental}

\subsection{Materials}

Glycol chitosan (GC, G7753), mercapto hexadecanoic acid (MHDA), N-hydroxysulfosuccinimide (NHS) and 1-Ethyl-3-[3dimethylaminopropyl]carbodiimide hydrochloride (EDC), 3-(4,5dimethylthiazol-2-yl)-2,5-diphenyl tetrazolium bromide (MTT), NADH, pyruvate, haemoglobin from bovine blood and Drabkin's reagent were acquired from Sigma-Aldrich. 5/6-Carboxyfluorescein succinimidyl ester was purchased from Thermo Scientific. Cell Culture reagents and culture medium were purchased from Biochrom.

\subsection{Nanogel self-assembling}

GC nanogel was synthesized and characterized as previously reported (Pereira et al., 2013). Briefly, GC nanogel was prepared by conjugation of the mercapto hexadecanoic acid (MHDA) to GC, through a carbodiimide reaction. Nanogel dispersions used in the different experiments were obtained after dispersing the lyophilized reaction product in distilled water, under magnetic stirring at $50{ }^{\circ} \mathrm{C}$ for $48 \mathrm{~h}$, and passed through a cellulose acetate syringe filter (pore size $0.45 \mu \mathrm{m}$ ).

\subsection{Cell cultures}

3T3 fibroblasts and mouse leukaemic monocyte macrophage (RAW 264.7) cell lines were maintained in DMEM supplemented with $10 \%$ bovine calf serum or fetal bovine serum (FBS), respectively, $100 \mathrm{IU} / \mathrm{mL}$ penicillin and $0.1 \mathrm{mg} / \mathrm{mL}$ streptomycin. Human microvascular endothelial cells (HMEC) were grown in RPMI1640 supplemented with 10\% FBS, Epidermal Growth Factor (EGF, $10 \mathrm{ng} / \mathrm{mL}$ ), Hydrocortisone $(1 \mu \mathrm{g} / \mathrm{mL}), 100 \mathrm{IU} / \mathrm{mL}$ penicillin and $0.1 \mathrm{mg} / \mathrm{mL}$ streptomycin. All cell lines were cultured as a monolayer in a humidified atmosphere containing $5 \% \mathrm{CO}_{2}$ at $37{ }^{\circ} \mathrm{C}$.

Murine Bone Marrow-Derived Macrophages were collected from femoral and tibial mouse bone marrow using a previously published protocol (Carvalho et al., 2011; Cerca et al., 2011). Briefly, mouse long bones were extracted from the mousse under aseptic conditions and flushed with RPMI-1640. The resulting cell suspension was centrifuged at $500 \mathrm{~g}$ during $10 \mathrm{~min}$. The cell pellet was resuspended in RPMI-1640 supplemented with 10 mM HEPES, $10 \%$ heat-inactivated FBS, $60 \mu \mathrm{g} / \mathrm{mL}$ penicillin/streptavidin, $0.005 \mathrm{mM} \beta$-mercaptoethanol (RPMI complete medium) and 10\% L929 Cell Conditioned Medium (LCCM). To remove adherent bone marrow cells, the cell suspension was incubated overnight at $37{ }^{\circ} \mathrm{C}$ and $5 \% \mathrm{CO}_{2}$ atmosphere in a Petri dish. The non-adherent cells were collected, centrifuged at $500 \mathrm{~g}(10 \mathrm{~min})$ and seeded in 24 well plates at $5 \times 10^{5}$ cells per well in RPMI complete medium containing $10 \%$ of $\mathrm{LCCM}$ and incubated at $37{ }^{\circ} \mathrm{C}$ in a $5 \% \mathrm{CO}_{2}$ atmosphere. Four days after seeding $10 \%$ of LCCM was re-added to the cultures. The culture medium was replaced with fresh RPMI complete medium containing $10 \%$ LCCM on day 7 . After 10 days in culture, cells were completely differentiated into macrophages.

\subsection{In vitro cell toxicity}

\subsubsection{MTT assay}

The cytotoxicity of GC nanogel was evaluated by using the quantitative colorimetric MTT assay. Cells were seeded onto 24-well cell culture plates at a density of $1 \times 10^{4}$ cells per well for 3 T3 and RAW and of $2 \times 10^{4}$ for HMEC, and left adhering in
$0.5 \mathrm{~mL}$ of culture medium overnight. Afterwards, the cells were incubated with nanogel dispersions at 0.1 and $0.5 \mathrm{mg} / \mathrm{mL}$ in fresh culture medium containing $25 \%$ of distilled water (v/v). Cells cultivated in medium with or without $25 \%$ of distilled water (v/v) (without nanogel) were used as controls. After 24, 48 and $72 \mathrm{~h}$ the metabolic activity was measured adding MTT (3-(4,5dimethylthiazol-2-yl)-2,5-diphenyl tetrazolium bromide). The tetrazolium salt is reduced by metabolically active cells using mitochondrial succinate dehydrogenase enzymes (Mosmann, 1983). The MTT solution ( $0.5 \mathrm{mg} / \mathrm{mL}$ in PBS) was carefully removed from each well and the resulting dark blue formazan crystals were solubilized in dimethyl sulfoxide and quantified spectrophotometrically at $570 \mathrm{~nm}$. The experiments were performed in triplicate and the metabolic activity results were shown as percentage of the values obtained at $0 \mathrm{~h}$ time point.

\subsection{2. $\mathrm{LDH}$ release assay}

Cytotoxicity can also be assessed by the degree of membrane damage. The $\mathrm{LDH}$ release assay measures the membrane integrity as function of the amount of cytoplasmic LDH leaked into the culture medium. The conversion of NADH and pyruvate into $\mathrm{NAD}^{+}$and lactate catalysed by LDH is the basis of the method (Rodrigues et al., 2012). Cells were seeded in 12-well plate at a density of the $2 \times 10^{5}$ cells per well for 3 T3 and HMEC and $1 \times 10^{5}$ for RAW and allowed to settle overnight in $0.5 \mathrm{~mL}$ of culture medium. The cells were treated with nanogel dispersions with a concentration of 0.1 and $0.5 \mathrm{mg} / \mathrm{mL}$, in fresh culture medium containing 25\% of distilled water $(\mathrm{v} / \mathrm{v})$. Untreated cells, exposed to $20 \%$ DMSO $(\mathrm{v} / \mathrm{v})$ or $25 \%$ distilled water $(\mathrm{v} / \mathrm{v})$ in culture media were used as controls. At the 24 and $48 \mathrm{~h}$ time points the culture medium from each well was collected and centrifuged at 13,000 rpm for $1 \mathrm{~min}$ and the cell free supernatant was collected and stored on ice for further extracellular LDH measurement. The respective cells were scraped with the aid of a Tris solution $15 \mathrm{mM}$ and lysed through sonication. Supernatants of centrifuged samples were used to quantify the intracellular LDH. Samples of extracellular $(40 \mu \mathrm{L})$ or intracellular $(10 \mu \mathrm{L}) \mathrm{LDH}$ were plated into a new microplate and $250 \mu \mathrm{L}$ of the NADH solution $0.31 \mathrm{mM}$ in phosphate buffer $0.05 \mathrm{M}$, pH7.4 added to each well. Finally, $10 \mu \mathrm{L}$ of a $8.96 \mathrm{mM}$ pyruvate solution in phosphate buffer (substrate solution) was added and immediately afterwards the variation of the absorbance at $340 \mathrm{~nm}$ was read in a microplate spectrophotometer, as to determine the rate of NADH consumption (slope of the line). LDH leakage was expressed as the ratio between extracellular and total $\mathrm{LDH}$, corresponding the inverse value to the cell membrane integrity. Untreated cells were used as a reference for the estimation of the maximum membrane integrity. Each experiment was performed in triplicate.

\subsection{Apoptosis assay}

The FITC Annexin V Apoptosis Detection Kit was used to determine apoptotic cell membrane changes in 3T3, HMEC and RAW cell lines. Cells $\left(2 \times 10^{5} /\right.$ well $)$ were seeded in a 12 -well plate and left adhering overnight. The cells were then incubated with nanogel dispersions at 0.1 and $0.5 \mathrm{mg} / \mathrm{mL}$ in fresh culture medium containing $25 \%$ of distilled water (v/v). A negative control assay was carried out without nanogel (the dilution with $25 \%$ of distilled water had no effect on the assay outcome), while $\mathrm{H}_{2} \mathrm{O}_{2}$ was used as positive control for apoptosis using different incubation times and concentrations according to the cell line $(0.5 \mathrm{mM}$ for $6 \mathrm{~h}-\mathrm{RAW}$; $0.2 \mathrm{mM}$ for $24 \mathrm{~h}-$ HMEC and $5 \mathrm{mM}$ for $3 \mathrm{~h}-3 \mathrm{~T} 3$ ) (Piao et al., 2011; Wang et al., 2007). After 24 h the cells were treated with $250 \mu \mathrm{L}$ trypsin/EDTA $0.25 \% / 0.02 \%$ in PBS for 2 min at $37^{\circ} \mathrm{C}$. The cell suspension was transferred to flow cytometry sample tubes (Beckman Coulter) and washed twice with cold PBS. Double staining with FITC-Annexin V/PI was performed as recommended by the 
supplier (BD Pharmigen) with minor modifications. In detail, each sample was incubated with $40 \mu \mathrm{L}$ of the work solution $(1.8 \mu \mathrm{L}$ of the Annexin V and PI diluted in $36.4 \mu \mathrm{L}$ of the annexin $\mathrm{V}$ binding buffer) for $15 \mathrm{~min}$ at room temperature, in the dark. Finally, $200 \mu \mathrm{L}$ of annexin $\mathrm{V}$ binding buffer was added to the samples that were then analysed by flow cytometry using a Coulter Epics XL Flow Cytometer (Beckman Coulter Inc., Miami, FL, USA).

\subsection{Cell cycle analysis}

To evaluate the effect of GC nanogel on cell cycle, 3T3 and RAW cells were seeded at a density of $5 \times 10^{4}$ cells per well, while HMEC cells were cultured at $1 \times 10^{5}$, in 6 -well plates, in $2 \mathrm{~mL}$ of the respective culture medium. In the following day, medium was replaced by GC nanogel dispersions with concentrations of 0.1 and $0.5 \mathrm{mg} / \mathrm{mL}$, in $2 \mathrm{~mL}$ of fresh culture medium containing $25 \%$ of distilled water $(\mathrm{v} / \mathrm{v})$. An additional condition without nanogel was carried out as a negative control (the dilution with $25 \%$ of distilled water had no effect on the assay). After $24 \mathrm{~h}$, cells were trypsinised and processed for flow cytometry analysis. In summary, the collected cells and the respective culture medium were centrifuged and washed with PBS. The cell suspension was kept on ice for $15 \mathrm{~min}$. Ice cold absolute ethanol was used to fix the cells during 15 min on ice. After washing with PBS to remove residual ethanol, the cells were treated with RNase A $(20 \mu \mathrm{g} / \mathrm{mL})$ during $15 \mathrm{~min}$ at $37{ }^{\circ} \mathrm{C}$. PI staining solution was added at least $15 \mathrm{~min}$ before sample analysis on the flow cytometer. Cell cycle progression of at least 20,000 single cells per sample was analysed by flow cytometry using a Coulter Epics XL Flow Cytometer (Beckman Coulter Inc., Miami, FL, USA) and analysed by using the FlowJo Analysis Software (Tree Star, Inc., Ashland, OR, USA).

\subsection{Complement activation}

Complement cascade was studied as reported previously (Lozano et al., 2011) and based on the NCL (Nanotechnology Characterization Laboratory) protocol for qualitative determination of total complement activation by Western blot analysis. Briefly, a pool of human plasma from healthy donors was incubated with $1 \mathrm{mg} / \mathrm{mL}$ of GC nanogel in the presence of veronal buffer. Equal volumes $(50 \mu \mathrm{L})$ of plasma, buffer and sample were mixed and incubated at $37^{\circ} \mathrm{C}$ for $60 \mathrm{~min}$. Cobra venom factor from Quidel Corporation (San Diego, CA, USA), and PBS were used as positive and negative controls, respectively. Proteins were resolved by $10 \%$ SDS-PAGE, and then transferred to a membrane (Immun-Blot PVDF Membrane, Biorad, Hercules, USA) using the transblot semidry BioRad transfer equipment (Trans blot SD, BioRad, Hercules, USA). The membranes were incubated for 90 min with a mouse monoclonal antibody against human C3 diluted 1:1000 (Abcam, Cambridge, UK), washed and incubation with secondary polyclonal goat anti-mouse IgG antibodies conjugated with alkaline phosphatase diluted 1:2000 (Dako, Glostrup, Denmark). The membrane was finally revealed with 5-Bromo-4-Chloro-3-Indolyl Phosphate (BCIP) (Sigma). For further analysis, membranes were scanned with ChemiDoc ${ }^{\mathrm{TM} X R S}+$ System (Bio-Rad; Hercules, CA). The percentage of the lower band was then quantified with Image Lab ${ }^{\text {TM }}$ Software 3.0.

\subsection{Confocal laser scanning microscopy}

GC nanogels were labelled with 5/6-carboxyfluorescein succinimidyl ester (NHS-Fluorescein), through an amide linkage, in order to study their cellular uptake by murine macrophages. In detail, the NHS-Fluorescein was dissolved in DMSO, at a concentration of $1 \%$. The molar ratio of NHS-Fluorescein carboxylic groups to the GC nanogel free amine groups was 0.25 . The dye was added to a stirred nanogel suspension at a concentration of $1 \mathrm{mg} / \mathrm{mL}$ in PBS. The reaction was allowed to occur overnight at room temperature, in the dark. The reaction mixture was thoroughly dialysed (MW cutoff $10-12 \mathrm{kDa}$ ) against distilled water to remove free NHSFluorescein. As to verify the absence of free dye, the final solution of NHS-Fluorescein labelled nanogel was purified by centrifugation through a $10 \mathrm{kDa}$ MW cutoff filter.

Murine macrophages were seeded at a density of $5 \times 10^{5}$ cells per well in a 24 well plate (with a coverslip in each well), and left adhering overnight. The cells were incubated with $0.2 \mathrm{mg} / \mathrm{mL}$ of each NHS-Fluorescein labelled nanogel suspension in culture medium containing $25 \%$ of water v/v. After $6 \mathrm{~h}$, the coverslips were washed twice with PBS at room temperature and the cells were fixed with paraformaldehyde $2 \%$ for $25 \mathrm{~min}$. After washing the cells twice with PBS twice, 4',6-diamidino-2-phenylindole (DAPI, $120 \mathrm{ng} /$ $\mathrm{mL}$ ) was used to stain the nucleus for $3 \mathrm{~min}$ at room temperature. After washing the preparations were observed in a confocal laser scanning microscope Leica SP2 AOBS SE (Leica Mycrosystems, Germany).

\subsection{Haemocompatibility studies}

The whole blood was collected from three independent healthy donors using citrated blood collection tubes.

\subsubsection{Haemolysis index}

The haemolysis assay was performed in agreement to the procedure described by the American Society for Testing Materials (ASTM F756-00, 2000), with slight modifications. Briefly, $0.5 \mathrm{~mL}$ of diluted blood at $10 \mathrm{mg} / \mathrm{mL}$ of haemoglobin concentration was added to $3.5 \mathrm{~mL}$ of the nanogel solution in PBS at $0.5 \mathrm{mg} / \mathrm{mL}$ and $0.1 \mathrm{mg} / \mathrm{mL}$ and incubated at $37^{\circ} \mathrm{C}$ for $3 \mathrm{~h}$. The tubes were gently inverted at each $30 \mathrm{~min}$ to homogenize the mixture. Ultrapure water and Phosphate-buffered saline (PBS) were used as positive and negative control, respectively. Then, the suspension was centrifuged at $750 \mathrm{~g}$ for $15 \mathrm{~min}$. $0.5 \mathrm{~mL}$ of the resulted supernatant was joined to a $0.5 \mathrm{~mL}$ Drabkin's reagent and left $15 \mathrm{~min}$ at room temperature. Finally, the absorbance was read at $540 \mathrm{~nm}$. The haemoglobin concentration of the freshly derived human blood was calculated using a calibration curve previously prepared with haemoglobin from bovine blood.

\subsubsection{Whole blood clotting time}

Whole blood kinetic clotting times of nanogel samples was performed as described by Leitao et al. (2013). Previously, the clotting was induced by addition of $10 \% 0.1 \mathrm{M} \mathrm{CaCl}_{2}$ to whole blood. The nanogel samples at 0.1 and $0.5 \mathrm{mg} / \mathrm{mL}$, glass microspheres (positive control) and 25\% v/v of PBS (negative control) in a 24-well polystyrene microtitter plate were incubated for $0,5,10,20$ and $30 \mathrm{~min}$ at room temperature with $150 \mu \mathrm{L}$ of activated whole blood. At the end of each time point, $3 \mathrm{~mL}$ of distilled water was added to the well and incubated for $5 \mathrm{~min}$, in order to lyse the red blood cells (and consequent haemoglobin release) that were not entrapped in the thrombus. $200 \mu \mathrm{L}$ of the supernatant was transferred to a 96-well polystyrene microtitter plate to measure the concentration of the released heamoglobin by spectrophotometric analysis at $540 \mathrm{~nm}$. All the samples were studied in triplicate.

\subsection{Statistical analysis}

The results were expressed as mean \pm SD of 3 independent experiments $(n=3)$. Statistical significances were performed applying $t$-test or two-way ANOVA tests through Prism software (GraphPad software version 5.00, USA). Significance of the results is indicated according to $P$ values with one, two and three of the 
used symbols $(*,+, \#)$ corresponding to $P<0.05, P<0.005$ and $P<0.0001$, respectively).

\section{Results}

\subsection{Cytotoxicity studies}

\subsubsection{Metabolic activity}

The cytotoxicity of the GC nanogel was assessed by MTT assay using a set of cell lines; slightly different results were obtained with each one (Fig. 1). The control with culture medium diluted with $25 \%$ of water presents a lower cell growth as compared to the straight medium, an effect probably due to the dilution of the nutrients. It must be remarked that in none of the tested nanogel concentrations the mitochondrial activity was lower than the one observed at time zero, indicating that the GC nanogel did not

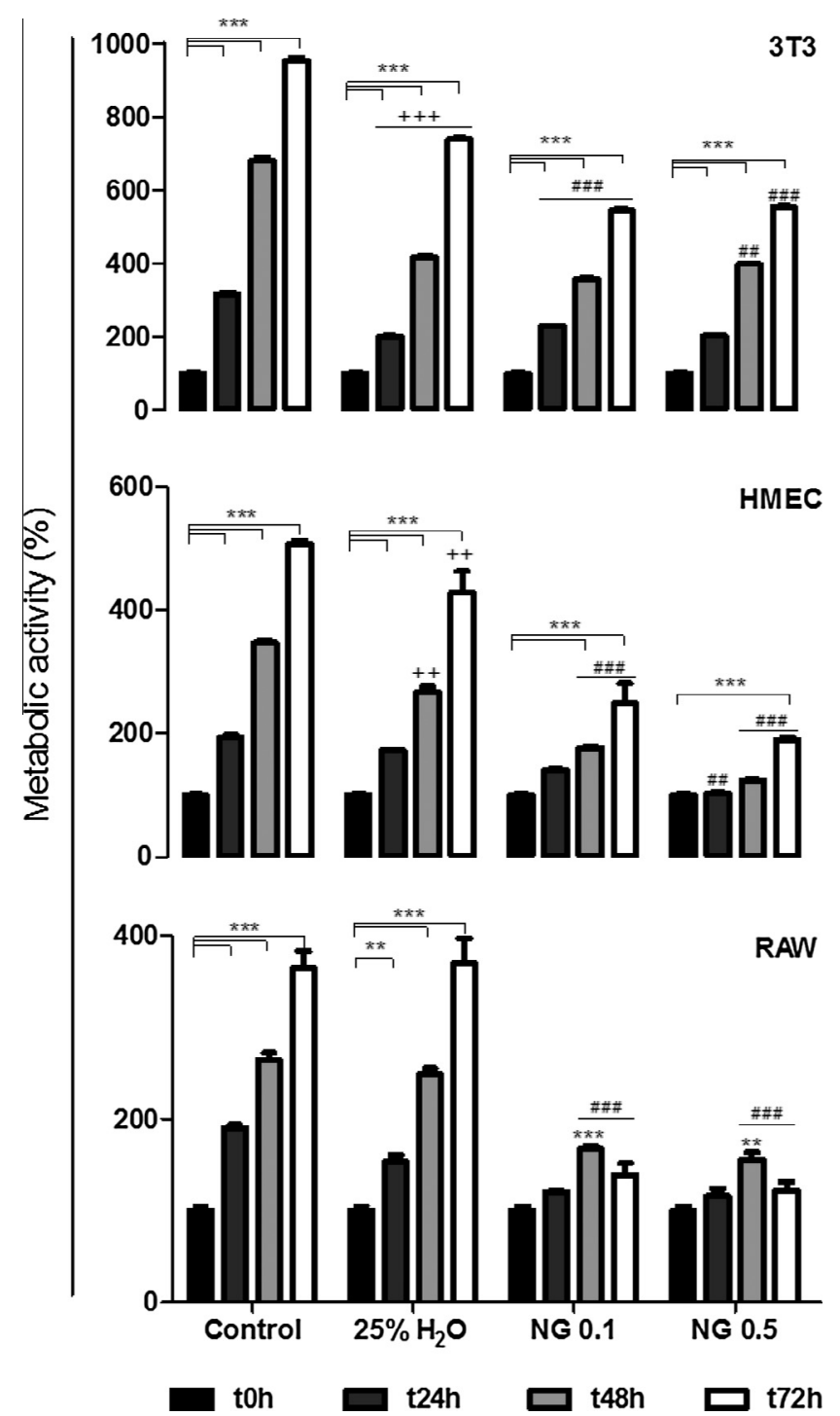

Fig. 1. Effect of GC nanogel at 0.1 and $0.5 \mathrm{mg} / \mathrm{mL}$ after at 24,48 and $72 \mathrm{~h}$ of incubation with 3T3, HMEC and RAW cell lines, assessed by MTT assay and expressed taking as reference the initial value. Statistical differences are determined through a 2-way ANOVA and represented by $(+)$ for the differences between cell culture medium (Control) and $25 \%$ of water content $(\mathrm{v} / \mathrm{v}$ ) into the well; whereas the differences between $25 \% \mathrm{H}_{2} \mathrm{O}$ and both concentrations of nanogel ( $\mathrm{NG} 0.1$ and NG 0.5) are shown as (\#); finally, concerning cell growth over the time, comparing $0 \mathrm{~h}$ incubation time with remaining incubation times for each condition, the statistical differences are presented as (*). induce cell death. On the other hand, all tested cell lines showed a lower metabolic activity in the presence of the nanogel when compared with the diluted medium control. A nanogel dose dependent effect on decreasing of cell proliferation rate was exhibited by HMEC (at 48 and $72 \mathrm{~h}$ incubation time), while no such correlation was observed for 3T3 an RAW cells. The HMEC and RAW cell lines were clearly more susceptible to nanogel treatment, mainly for later incubation times.

\subsubsection{Cell membrane integrity}

The potential cytotoxicity of the GC nanogel was also evaluated by measuring the cell membrane integrity through LDH leakage quantification. As shown in Fig. 2, the GC nanogel in concentrations of 0.1 and $0.5 \mathrm{mg} / \mathrm{mL}$ did not affect the cell membrane integrity, since a significant $\mathrm{LDH}$ release to the culture medium was observed only in the positive control (20\% of DMSO).

\subsection{Apoptosis assay}

Annexin V FITC/propidium iodide dual staining was used to investigate the influence of GC nanogel on cell death induction. This assay is based on the observation since early stages of apoptosis of the phosphatidylserine translocation to the external surface of the plasma membrane. Annexin $\mathrm{V}$ was used as a sensitive probe

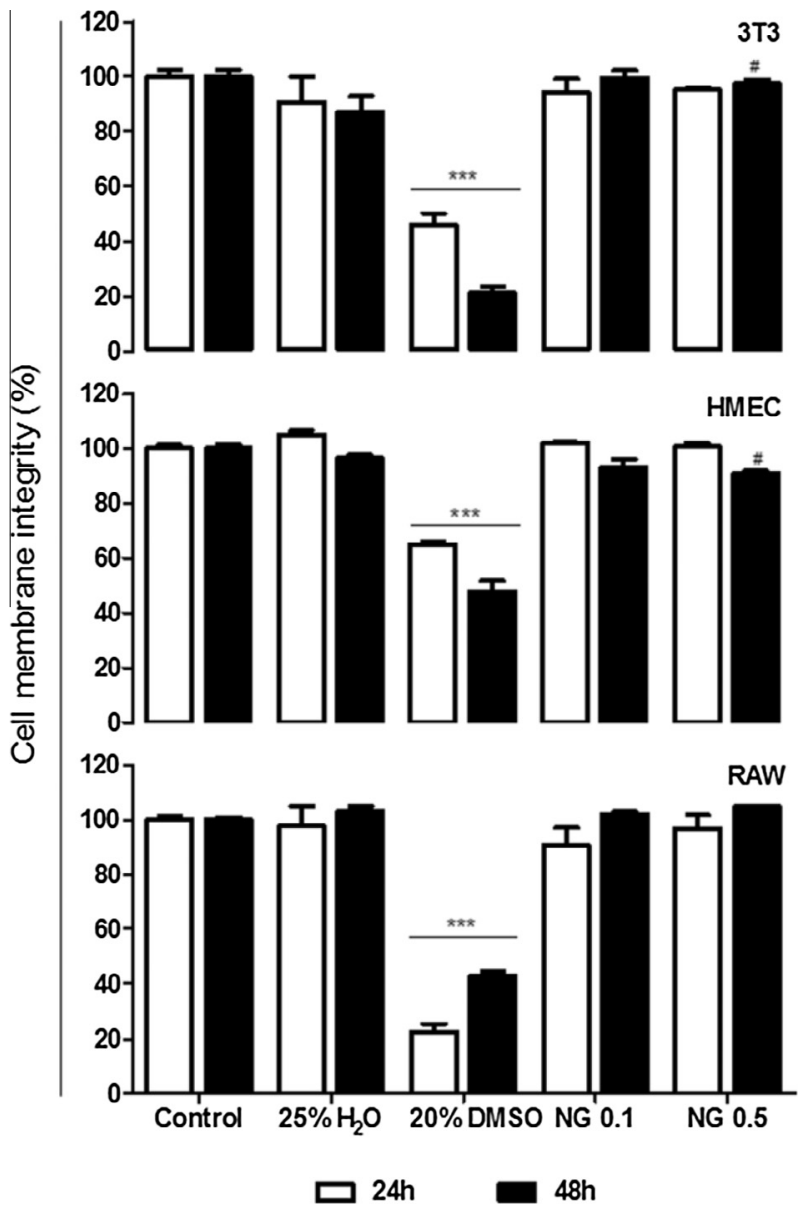

Fig. 2. Effect of GC nanogel at 0.1 and $0.5 \mathrm{mg} / \mathrm{mL}$ after 24 and $48 \mathrm{~h}$ of incubation with 3T3, HMEC and RAW cells on the amount of LDH released to culture medium. Cell culture medium and $20 \%$ of DMSO were used as negative and positive control, respectively. Statistical differences were found by a $t$-test in each time of incubation between negative control and $25 \% \mathrm{H}_{2} \mathrm{O}$ or $20 \%$ DMSO are shown as (*); while differences between $25 \% \mathrm{H}_{2} \mathrm{O}$ and nanogel samples (NG 0.1 and $\mathrm{NG} 0.5$ ) are represented as (\#). 
since it is a calcium-dependent phospholipid binding protein, with high affinity to phosphatidylserine. Cells in later stages of apoptosis $\left(\right.$ Annexin $\mathrm{V}^{+} / \mathrm{PI}^{+}$) or necrosis (Annexin $\mathrm{V}^{-} / \mathrm{PI}^{+}$) were identified through detection of PI binding to nuclear DNA allowed by loss of cell membrane integrity (Koopman et al., 1994; Vermes et al., 1995). Viable and early apoptotic cells were recognized through Annexin $\mathrm{V}^{-} / \mathrm{PI}^{-}$and Annexin $\mathrm{V}^{+} / \mathrm{PI}^{-}$staining pattern, respectively. As shown in Fig. 3, the percentage of apoptotic and late-apoptotic cells incubated with the nanogel was not significantly different from the negative control, exception made for the HMEC cell line, which displays a slight increase in the Annexin positive cells. A dose dependent effect on apoptosis induction was indeed observed in this case.

\subsection{Effect of the nanogel on cell cycle arrest}

Cell cycle analysis was performed to ascertain whether cell growth inhibition observed in the MTT assay was correlated with cell cycle arrest, since no effect on cell death by apoptosis/necrosis was verified. The distribution of cell cycle phases of 3T3, HMEC and RAW cells treated with nanogel at 0.1 and $0.5 \mathrm{mg} / \mathrm{mL}$ for $24 \mathrm{~h}$ is shown on Fig. 4A. As could be observed no effect on HMEC and 3T3 cell cycle progression was verified, except for the 3T3 population incubated with the higher nanogel concentration, where a slight decrease of the G2 phase was observed. However, in RAW cells, a highly significant dose dependent cell cycle arresting was verified on G1 phase. This increase of G1 population is correlated with a reduction of the $S$ and $G 2$ phases, as may be observed on the cell cycle distribution presented on Fig. 4B.

\subsection{Evaluation of complement activation}

In this assay the cleavage product of the $\mathrm{C} 3$ component was used as a marker for complement activation by any pathway. Therefore, by studying the degradation of the $\mathrm{C} 3$ factor we can determine whether the nanogels have a potential effect on the complement activation cascade. The western blot assay for the C3 fragment detection was performed after incubation of the GC nanogel $(1 \mathrm{mg} / \mathrm{mL})$ with human plasma. The results are shown in Fig. 5A. The upper band of $115 \mathrm{kDa}$ corresponds to the intact C3 factor and the one with $43 \mathrm{kDa}$ to the main degradation product. The protein degradation was quantified considering the intensity of the band at $43 \mathrm{kDa}$ normalized to the value obtained with the positive control (cobra venom factor). As could be observed in Fig. 5B the percentage of $\mathrm{C} 3$ cleavage product(s) was similar to those found in the negative control, so it may be concluded that the GC nanogel does not activate the complement system.

\subsection{Murine macrophages cellular uptake}

In order to investigate whether the nanogels are phagocytosed by macrophages, fluorescent nanogels were incubated with murine macrophages. Fig. 6A and B illustrate the cellular uptake of FITC labelled dextrin nanogel (used as a positive control of macrophages uptake, as shown by Gonçalves et al. and NHS-Fluorescein labelled GC nanogels, respectively (Goncalves et al., 2010). Interestingly, the GC nanogel was poorly internalized by murine macrophages as compared with dextrin nanoparticles.

\subsection{Haemocompatibility studies}

\subsubsection{Haemolysis index}

According to the Standard Practice for Assessment of Haemolytic Properties of Materials from the American Society for Testing Materials (ASTM F756-00, 2000) the nanogel was non-haemolytic at the concentrations tested, since the haemolytic index is inferior to
5\% (Table 1), although a slight effect is observed for the higher concentration.

\subsubsection{Whole blood clotting time}

In this assay, human whole blood was allowed to clot in contact with the GC nanogel to assess the nanomaterial likelihood to be thrombogenic in vivo. As clotting occurs, more red blood cells are retained in the clot, and therefore less haemoglobin is released by lysis upon addition of distilled water. The results (Fig. 7) showed that the nanogel samples exhibited a similar behaviour to the negative control (PBS), thus being classified as nonthrombogenic; conversely, as expected, the glass microspheres showed the quickest clotting time.

\section{Discussion}

Although GC is recognized as a highly biocompatible chitosan derivative (Carreño-Gómez and Duncan, 1997), a definitive statement on this matter requires further experimental evidences. Thus, the purpose of the present work was to perform a comprehensive study of the GC based-nanogel biocompatibility. The GC used for nanogel synthesis, purchase from Sigma-Aldrich, were thoroughly characterized in our previous work, due to the lack of consistent data in literature. The amphiphilic polymer resultant from covalent attachment of hydrophobic chains on the hydrophilic GC backbone $(100 \mathrm{kDa})$ self-assembles in water medium originating nanogels with an average size of the $250 \mathrm{~nm}$ and positive surface charge (+30 mV). (Pereira et al., 2013).

The metabolic activity studies show that the GC nanogel does note reduce the mitochondrial activity overtime; however, cell growth inhibition was observed in all cell lines. The HMEC cell proliferation reduction (at 48 and $72 \mathrm{~h}$ incubation time) was found to be dose dependent, while no such correlation was observed for 3T3 an RAW cells. Anitha et al. reported a similar dose independent effect observed in two cell lines of fibroblasts (L929, NIH3T3) after $48 \mathrm{~h}$ exposure to thiolated chitosan nanoparticles (Anitha et al., 2011). Absence of cytotoxicity and cell growth inhibition effect in L929 fibroblasts treated with chitosan-g-poly(N-vinylcaprolactam) nanoparticles was reported by Rejinold et al. after $24 \mathrm{~h}$ of incubation (Rejinold et al., 2011). The same was observed in this work for the 3T3 cell line, after short incubation period $(24 \mathrm{~h})$. The HMEC and RAW cell lines were clearly more susceptible to nanogel treatment, mainly for later incubation periods. Equivalent RAW cell growth inhibition rate was obtained by Kim et al. for NCTC 3749 macrophage cells (20-30\%) treated with $0.1 \mathrm{mg} / \mathrm{mL}$ of mannosylated nanoparticles for $24 \mathrm{~h}$ (Kim et al., 2006). It is noteworthy that fairly high concentrations of the nanogel were used in the current study, purposely to detect toxic effects. Applications of the biomaterial are not likely to reach such concentrations in vivo, nor the contact time with cells will be as long as the tested ones; hence the results achieved can be considered indicative of the safety of the material. The absence of the GC nanogel cytotoxicity was also confirmed by measuring the cell membrane integrity through LDH leakage quantification. According to Fotakis and Timbrell (2006), the LDH leakage assay is not as sensitive as the MTT assay for cytotoxicity detection, since it requires higher concentration of the sample or longer incubation time. Although being a cell-type dependent method, we did not observe significant differences by increasing the incubation time or varying the sample concentration. The apoptosis assay corroborate that GC nanogel did not induce cell death by necrosis and/or apoptosis, in agreement with LDH release results, since necrosis would result in the loss of cell membrane integrity (Vermes et al., 1995). Moreover, the cell cycle analysis demonstrated that any effect on HMEC and 3T3 cell cycle progression was verified. However, in RAW cells a highly 
(A)
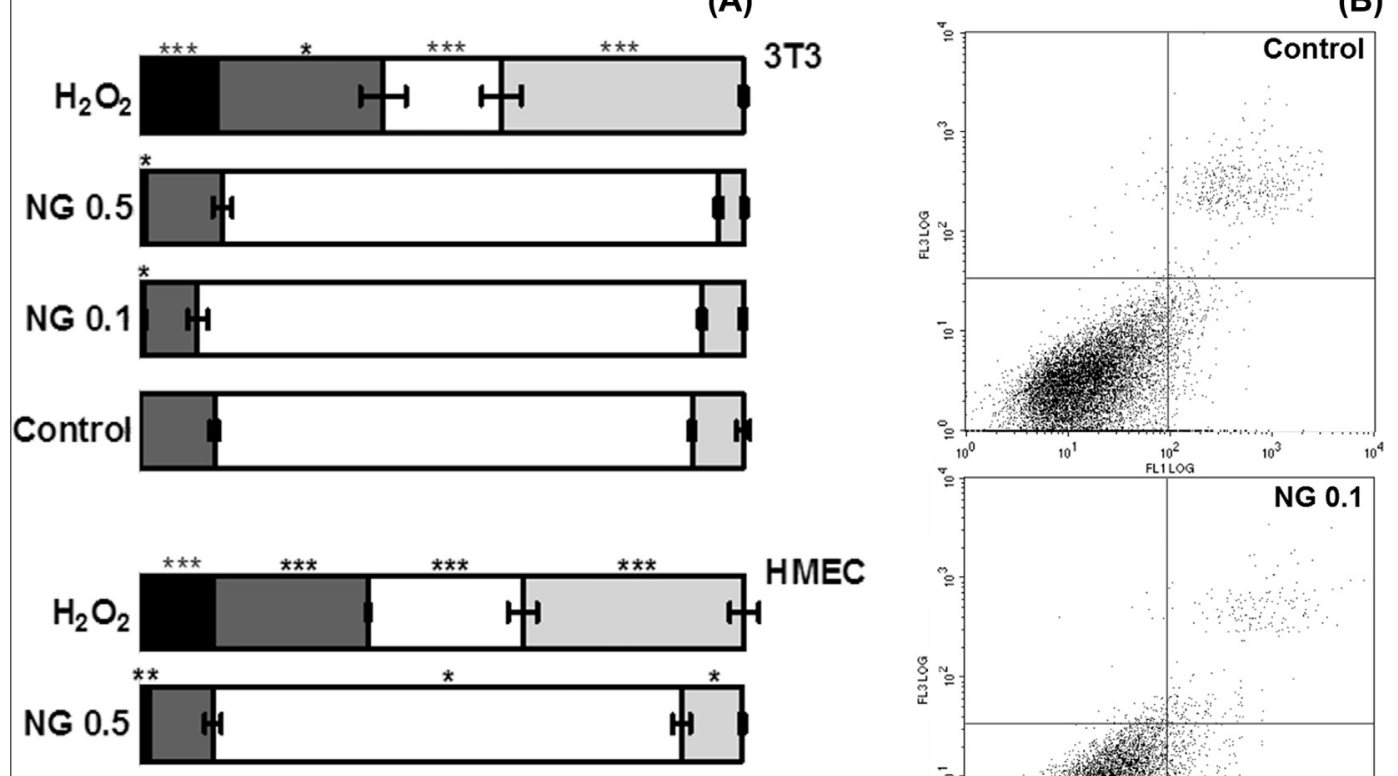

HMEC
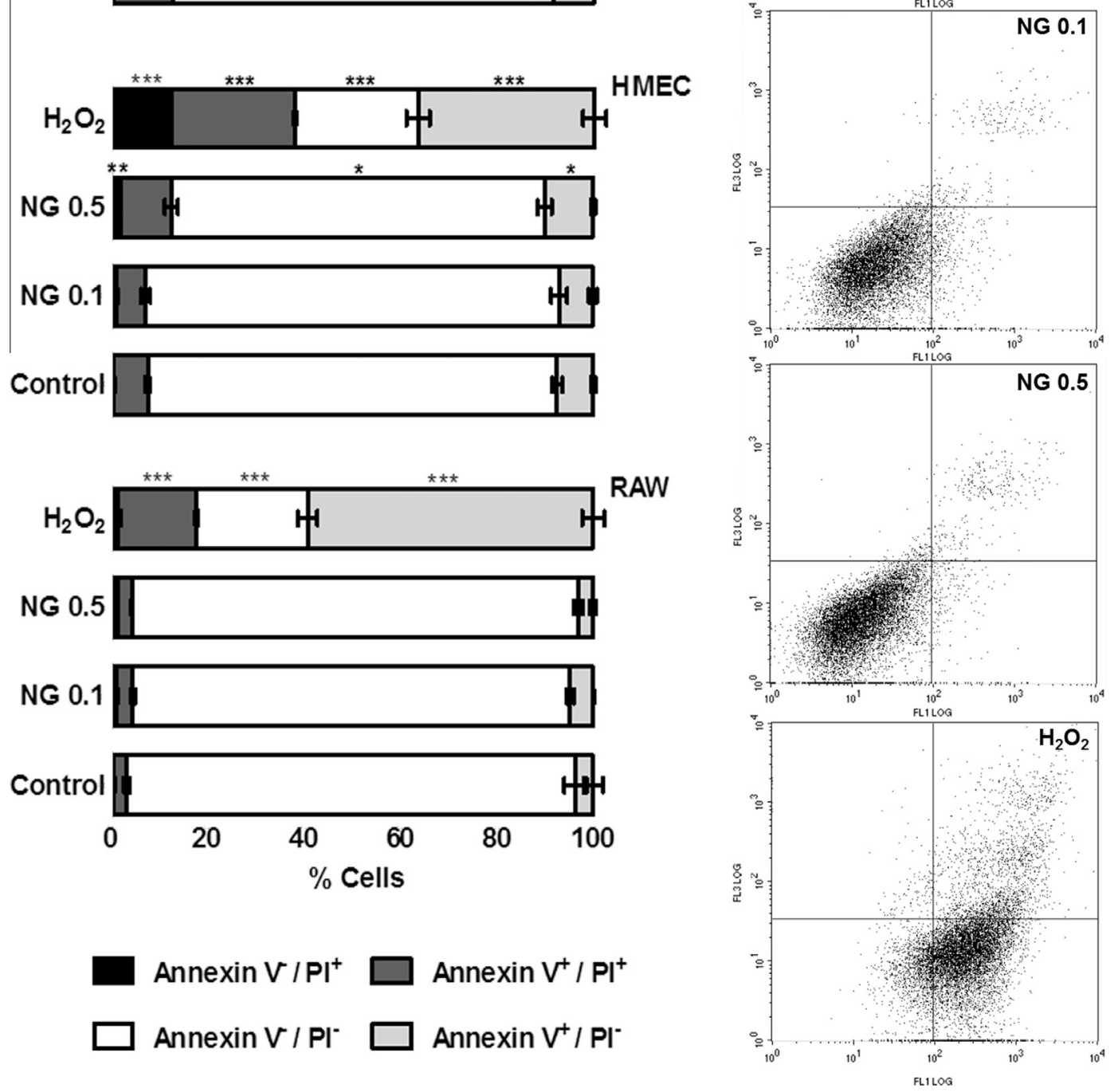

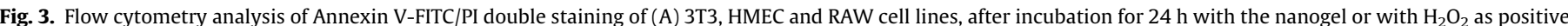

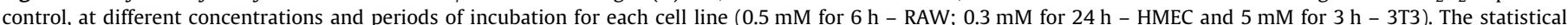

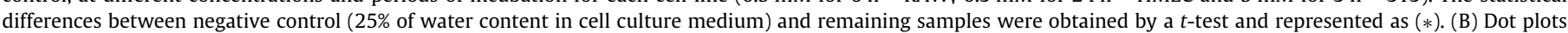

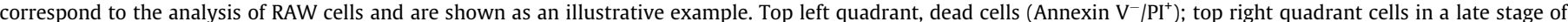
apoptosis (Annexin $\mathrm{V}^{+} / \mathrm{PI}^{+}$); bottom right quadrant, apoptotic cells (Annexin $\mathrm{V}^{+} / \mathrm{PI}^{-}$) and bottom left quadrant, viable cells $\left(\right.$Annexin $\mathrm{V}^{-} / \mathrm{PI}^{-}$).

significant dose dependent cell cycle arresting was found on G1 phase, which may be due to phagocytic activity, and therefore higher concentration of the nanogel inside the cells. Nevertheless, as shown above bone marrow derived macrophages internalize GC nanogel to little extent. Similar studies on the effect of drug loaded GC nanoparticles on the cell cycle arresting have been reported. Surprisingly, these studies usually just show the effect of the drug, and no data regarding to the effect of the nanocarrier alone could be found (Park et al., 2006).
Depending on the nanoparticles administration pathways and their physicochemical properties, they could induce different immune reactions that are initiated with adsorption of opsonins to the nanoparticles surface, triggering the complement system. By itself, opsonization is not able to destroy the invaders but it acts as a border guard priming the surface of nanoparticles for rapid recognition and clearance by complement receptor bearing cells such as blood monocytes and macrophages of the mononuclear phagocyte system (MPS). (Denis, 2012; Peter and Moghimi, 2012) 

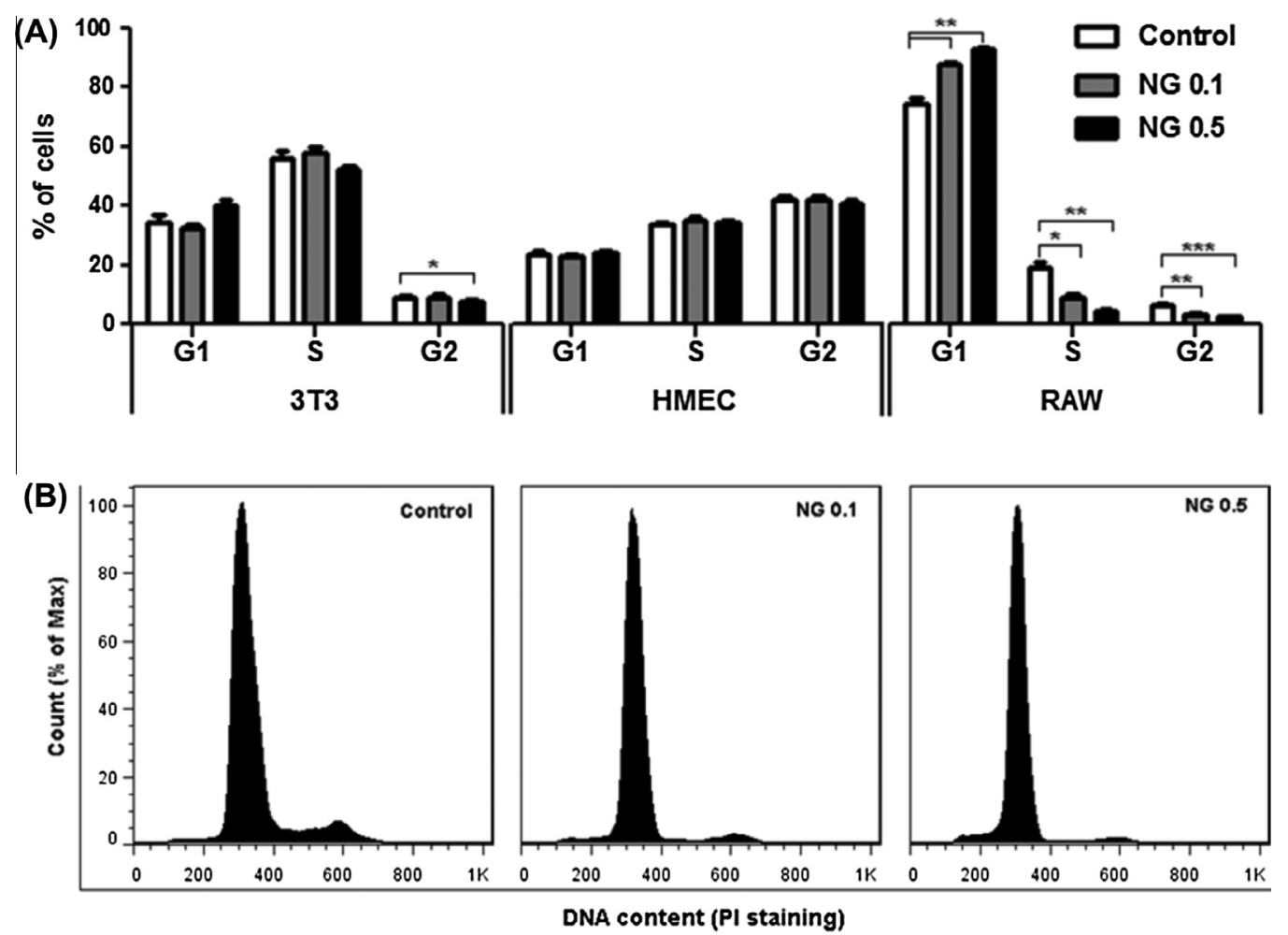

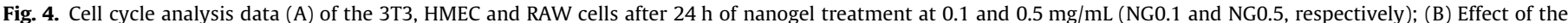

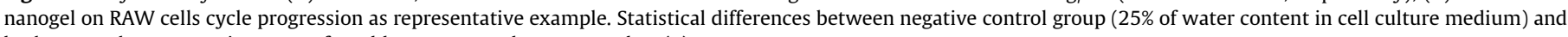
both nanogel concentrations were found by a $t$-test and represented as $(*)$.
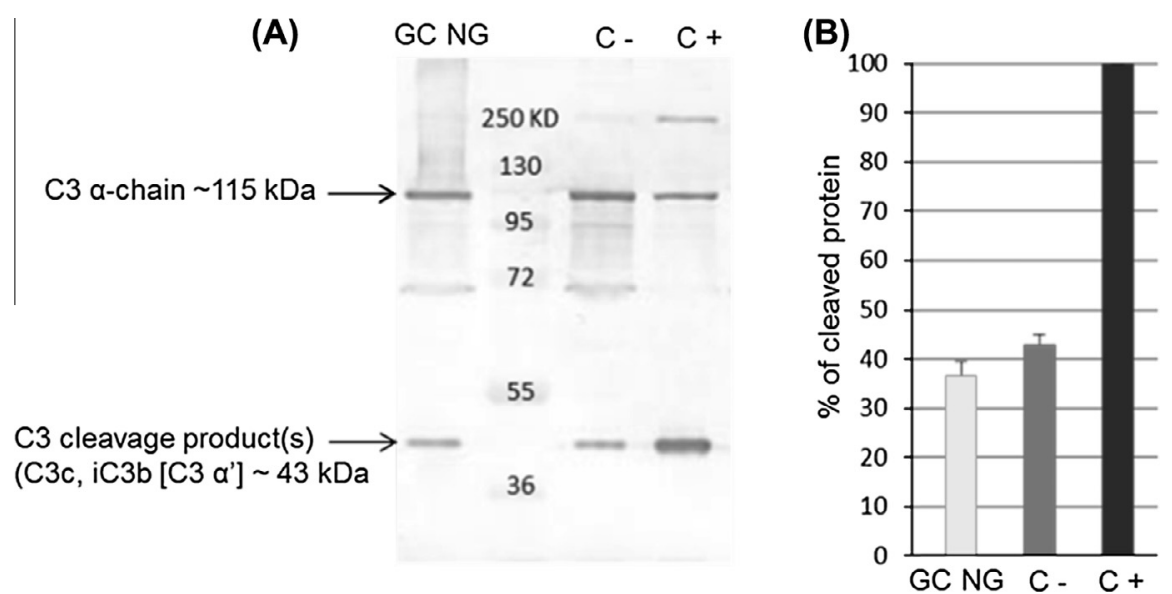

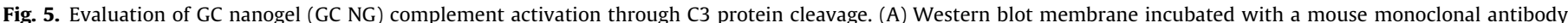

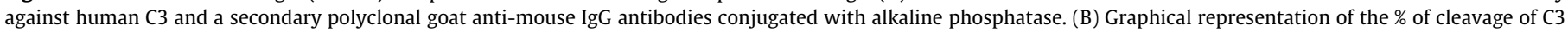
protein induced by GC nanogel (GC NG) as compared to negative (C-) and positive ( $\left.\mathrm{C}^{+}\right)$controls (PBS and Cobra venom factor, respectively).

Regardless the initial events that determine which pathway leads to complement activation, all converge to the cleavage of the central component C3 (Carroll, 2004). So, as human serum in the presence of the GC nanogel revealed similar percentages of C3 cleavage product(s) to those found in the negative control, could be concluded that the GC nanogel does not activate the complement system. Indeed, Bertholon et al. refers chitosan as a weaker activator of the complement cascade. They showed that increasing the molecular weight of chitosan led to decreased complement activation, reaching negligible levels. (Bertholon et al., 2006) Marchand et al. also concluded that chitosan is a non-activating biomaterial; in spite of binding anionic plasma and serum proteins like $\mathrm{C} 3$, it does not led to complement activation (Marchand et al., 2010). As the long systemic circulation half-life of the nanoparticles is dependent on their ability to avoid the capture by macrophages of the MPS, the development of 'stealth' nanoparticles is highly desirable. (Fang et al., 2012) Interestingly, we found that GC nanogel was poorly internalized by bone marrow derived macrophages as compared with dextrin nanoparticles. (Goncalves et al., 2010) Sarmento et al. also reported that chitosan coated solid lipid nanoparticles were neglectfully internalized within RAW 264.7 cells, as compared with uncoated solid lipid nanoparticles (Sarmento et al., 2011). Indubitably this is a promising result since GC nanogel may thus evade blood clearance and keep on circulation enough time to find the target site.

Charged particles readily interact with negatively charged cell surface; probably, they can do so with negatively charged red blood cells (RBCs). (Sarkar et al., 2013) Therefore, we investigated 

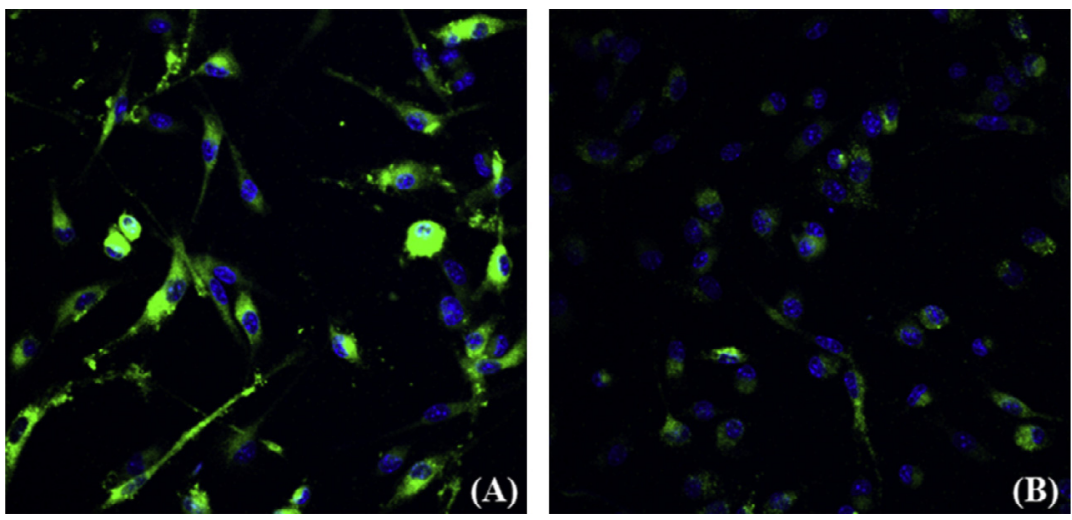

Fig. 6. Confocal microscopy images of murine bone marrow derived macrophages treated with fluorescent labelled (A) dextrin and (B) GC nanogels for 6 h.

Table 1

Blood haemolysis index after treatment with nanogel samples for $3 \mathrm{~h}$ at $37^{\circ} \mathrm{C}$

\begin{tabular}{lc}
\hline Sample & Haemolytic index (\%) \\
\hline GC nanogel $0.5 \mathrm{mg} / \mathrm{mL}$ & $3.183 \pm 0.137$ \\
GC nanogel $0.1 \mathrm{mg} / \mathrm{mL}$ & $1.423 \pm 0.366$ \\
C+ (ultrapure water) & $98.773 \pm 0.816$ \\
\hline
\end{tabular}

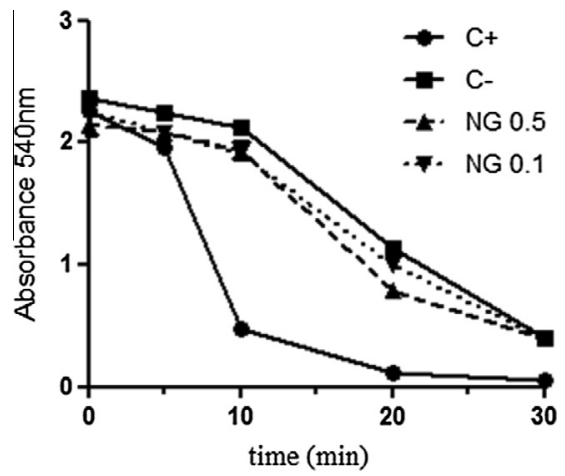

Fig. 7. Whole blood clotting time for nanogel samples at 0.1 and $0.5 \mathrm{mg} / \mathrm{mL}$. The positive control $(\mathrm{C}+)$ used were glass microspheres and the negative (C-) PBS. No statistical differences were observed between negative control and nanogel samples.

the release of haemoglobin from RBCs. The GC nanogel was found to be non-hemolytic, even though both haemolytic and nonhaemolytic effects have been previously assigned to chitosan based nanoparticles (Layek and Singh, 2013; Nogueira et al., 2013). The GC nanogel was found to be non-haemolytic, and may also be considered non-thrombogenic, in agreement with He et al., who have found that the positive charge of chitosan retarded thrombin generation and blood coagulation. (He et al., 2013).

\section{Conclusion}

The biocompatibility of GC nanogels was comprehensively characterized. Although slightly reducing the growth rate of RAW and HMEC cell lines such an effect could not be explained by cell membrane compromising nor apoptosis/necrosis induction. A more severe effect observed in the case of RAW cells could be at least partially explained by the observed effect of cell cycle arrest; however for the HMEC this was not the case, an issue that remains to be clarified. It must be remarked that fairly high concentrations of the nanogel were used in these assays. Under physiological conditions associated to a possible application of this material, lower concentrations and contact times should arise, such that we may classify the GC nanogel as safe. In conclusion, the present study demonstrate that the GC nanogel is able to be systemically administered, since it did not activate the complement system, evade the MPS, did not interact with RBC's and was found to be non-thrombogenic. These findings indicate that GC nanogel is a promising biocompatible vehicle for drug delivery.

\section{Conflict of Interest}

The authors declare that there are no conflicts of interest.

\section{Transparency Document}

The Transparency document associated with this article can be found in the online version.

\section{Acknowledgments}

Paula Pereira thanks FCT, the Ph.D. grant ref SFRH/BD/64977/ 2009. This work was also supported by a grant from the Spanish Ministry of Economy and Competitivity (SAF2011-30337-C0202). We also acknowledge the European Union Seventh Framework Programme [FP7/REGPOT-2012-2013.1] under grant agreement BIOCAPS-316265. MP acknowledges fellowship from Spanish Ministry of Education (FPU predoctoral grant program).

\section{References}

Anitha, A., Deepa, N., Chennazhi, K.P., Nair, S.V., Tamura, H., Jayakumar, R., 2011 Development of mucoadhesive thiolated chitosan nanoparticles for biomedical applications. Carbohydr. Polym. 83, 66-73.

Bernkop-Schnurch, A., Dunnhaupt, S., 2012. Chitosan-based drug delivery systems. Eur. J. Pharm. Biopharm. 81, 463-469.

Bertholon, I., Vauthier, C., Labarre, D., 2006. Complement activation by core-shell poly(isobutylcyanoacrylate)-polysaccharide nanoparticles: influences of surface morphology, length, and type of polysaccharide. Pharm. Res. 23, 1313-1323.

Carreño-Gómez, B., Duncan, R., 1997. Evaluation of the biological properties of soluble chitosan and chitosan microspheres. Int. J. Pharm. 148, 231-240.

Carroll, M.C., 2004. The complement system in regulation of adaptive immunity. Nat. Immunol. 5, 981-986.

Carvalho, V., Castanheira, P., Madureira, P., Ferreira, S.A., Costa, C., Teixeira, J.P., Faro, C., Vilanova, M., Gama, M., 2011. Self-assembled dextrin nanogel as protein carrier: controlled release and biological activity of IL-10. Biotechnol. Bioeng. 108, 1977-1986.

Cerca, F., Andrade, F., Franca, A., Andrade, E.B., Ribeiro, A., Almeida, A.A., Cerca, N., Pier, G., Azeredo, J., Vilanova, M., 2011. Staphylococcus epidermidis biofilms with higher proportions of dormant bacteria induce a lower activation of murine macrophages. J. Med. Microbiol. 60, 1717-1724.

Croisier, F., Jérôme, C., 2013. Chitosan-based biomaterials for tissue engineering. Eur. Polymer J. 49, 780-792. 
Denis, L., 2012. The interactions between blood and polymeric nanoparticles depend on the nature and structure of the hydrogel covering the surface. Polymers, 4

Fang, R.H., Hu, C.M., Zhang, L., 2012. Nanoparticles disguised as red blood cells to evade the immune system. Exp. Opin. Biol. Ther. 12, 385-389.

Fotakis, G., Timbrell, J.A., 2006. In vitro cytotoxicity assays: comparison of LDH, neutral red, MTT and protein assay in hepatoma cell lines following exposure to cadmium chloride. Toxicol. Lett. 160, 171-177.

Gaspar, R., Duncan, R., 2009. Polymeric carriers: preclinical safety and the regulatory implications for design and development of polymer therapeutics. Adv. Drug Deliv. Rev. 61, 1220-1231.

Goncalves, C., Torrado, E., Martins, T., Pereira, P., Pedrosa, J., Gama, M., 2010. Dextrin nanoparticles: studies on the interaction with murine macrophages and blood clearance. Colloids Surf. B Biointerfaces 75, 483-489.

He, Q., Gong, K., Ao, Q., Ma, T., Yan, Y., Gong, Y., Zhang, X., 2013. Positive charge of chitosan retards blood coagulation on chitosan films. J. Biomater. Appl. 27, 1032-1045.

Kim, T.H., Jin, H., Kim, H.W., Cho, M.H., Cho, C.S., 2006. Mannosylated chitosan nanoparticle-based cytokine gene therapy suppressed cancer growth in BALB/c mice bearing CT-26 carcinoma cells. Mol. Cancer Ther. 5, 1723-1732.

Koopman, G., Reutelingsperger, C.P., Kuijten, G.A., Keehnen, R.M., Pals, S.T., van Oers, M.H., 1994. Annexin V for flow cytometric detection of phosphatidylserine expression on B cells undergoing apoptosis. Blood 84, 1415-1420.

Layek, B., Singh, J., 2013. Amino acid grafted chitosan for high performance gene delivery: comparison of amino acid hydrophobicity on vector and polyplex characteristics. Biomacromolecules 14, 485-494.

Leitao, A.F., Gupta, S., Silva, J.P., Reviakine, I., Gama, M., 2013. Hemocompatibility study of a bacterial cellulose/polyvinyl alcohol nanocomposite. Colloids Surf. B Biointerfaces 111C, 493-502.

Liu, Y., Kong, M., Feng, C., Yang, K.K., Li, Y., Su, J., Cheng, X.J., Park, H.J., Chen, X.G., 2013. Biocompatibility, cellular uptake and biodistribution of the polymeric amphiphilic nanoparticles as oral drug carriers. Colloids Surf. B Biointerfaces 103, 345-353.

Lozano, T., Rey, M., Rojas, E., Moya, S., Fleddermann, J., Estrela-Lopis, I., Donath, E., Wang, B., Mao, Z., Gao, C., González-Fernández, Á., 2011. Cytotoxicity effects of metal oxide nanoparticles in human tumor cell lines. J. Phys: Conf. Ser. 304, 012046.

Marchand, C., Bachand, J., Perinet, J., Baraghis, E., Lamarre, M., Rivard, G.E., De Crescenzo, G., Hoemann, C.D., 2010. C3, C5, and factor B bind to chitosan without complement activation. J. Biomed. Mater. Res. A 93, 1429-1441.
Mosmann, T., 1983. Rapid colorimetric assay for cellular growth and survival: application to proliferation and cytotoxicity assays. J. Immunol. Methods 65, 55-63.

Naahidi, S., Jafari, M., Edalat, F., Raymond, K., Khademhosseini, A., Chen, P., 2013. Biocompatibility of engineered nanoparticles for drug delivery. J. Control. Release 166, 182-194.

Nogueira, D.R., Tavano, L., Mitjans, M., Perez, L., Infante, M.R., Vinardell, M.P., 2013 In vitro antitumor activity of methotrexate via pH-sensitive chitosan nanoparticles. Biomaterials 34, 2758-2772.

Park, J.S., Han, T.H., Lee, K.Y., Han, S.S., Hwang, J.J., Moon, D.H., Kim, S.Y., Cho, Y.W. 2006. N-acetyl histidine-conjugated glycol chitosan self-assembled nanoparticles for intracytoplasmic delivery of drugs: endocytosis, exocytosis and drug release. J. Control. Release 115, 37-45.

Pereira, P., Morgado, D., Crepet, A., David, L., Gama, F.M., 2013. Glycol chitosanbased nanogel as a potential targetable carrier for siRNA. Macromol. Biosci. 13, 1369-1378.

Peter, P.W., Moghimi, S.M., 2012. Complement sensing of nanoparticles and nanomedicines, functional nanoparticles for bioanalysis, nanomedicine, and bioelectronic devices volume 2 . Am. Chem. Soc., 365-382

Piao, S., Cha, Y.N., Kim, C., 2011. Taurine chloramine protects RAW 264.7 macrophages against hydrogen peroxide-induced apoptosis by increasing antioxidants. J. Clin. Biochem. Nutr. 49, 50-56.

Rejinold, N.S., Chennazhi, K.P., Nair, S.V., Tamura, H., Jayakumar, R., 2011 Biodegradable and thermo-sensitive chitosan-g-poly(N-vinylcaprolactam) nanoparticles as a 5-fluorouracil carrier. Carbohydr. Polym. 83, 776-786.

Rodrigues, S., Dionísio, M., López, C.R., Grenha, A., 2012. Biocompatibility of chitosan carriers with application in drug delivery. J. Functional Biomater. 3, 615-641.

Sarkar, K., Chatterjee, A., Chakraborti, G., Kundu, P.P., 2013. Blood compatible Nmaleyl chitosan-graft-PAMAM copolymer for enhanced gene transfection. Carbohydr. Polym. 98, 596-606.

Sarmento, B., Mazzaglia, D., Bonferoni, M.C., Neto, A.P., do Céu Monteiro, M., Seabra V., 2011. Effect of chitosan coating in overcoming the phagocytosis of insulin loaded solid lipid nanoparticles by mononuclear phagocyte system. Carbohydr. Polym. 84, 919-925.

Vermes, I., Haanen, C., Steffens-Nakken, H., Reutelingsperger, C., 1995. A novel assay for apoptosis. Flow cytometric detection of phosphatidylserine expression on early apoptotic cells using fluorescein labelled Annexin V. J. Immunol. Methods 184, 39-51.

Wang, J., Shen, W.H., Jin, Y.J., Brandt-Rauf, P.W., Yin, Y., 2007. A molecular link between E2F-1 and the MAPK cascade. J. Biol. Chem. 282, 18521-18531. 